\title{
Anderson Photon-Phonon Colocalization in Certain Random Superlattices
}

\author{
G. Arregui, ${ }^{1,2, *}$ N. D. Lanzillotti-Kimura, ${ }^{3}$ C. M. Sotomayor-Torres, ${ }^{1,4}$ and P. D. García ${ }^{1, \dagger}$ \\ ${ }^{1}$ Catalan Institute of Nanoscience and Nanotechnology (ICN2), CSIC and BIST, \\ Campus UAB, Bellaterra, 08193 Barcelona, Spain \\ ${ }^{2}$ Dept. de Física, Universitat Autnònoma de Barcelona, 08193 Bellaterra, Spain \\ ${ }^{3}$ Centre de Nanosciences et de Nanotechnologies (C2N), CNRS, Univ. Paris-Sud, Université Paris-Saclay, \\ 10 Boulevard Thomas Gobert, 91120 Palaiseau, France \\ ${ }^{4}$ Institució Catalana de Recerca i Estudis Avançats (ICREA), 08010 Barcelona, Spain
}

(Received 1 June 2018; revised manuscript received 30 November 2018; published 1 February 2019)

\begin{abstract}
Fundamental observations in physics ranging from gravitational wave detection to laser cooling of a nanomechanical oscillator into its quantum ground state rely on the interaction between the optical and the mechanical degrees of freedom. A key parameter to engineer this interaction is the spatial overlap between the two fields, optimized in carefully designed resonators on a case-by-case basis. Disorder is an alternative strategy to confine light and sound at the nanoscale. However, it lacks an a priori mechanism guaranteeing a high degree of colocalization due to the inherently complex nature of the underlying interference processes. Here, we propose a way to address this challenge by using GaAs/AlAs vertical distributed Bragg reflectors with embedded geometrical disorder. Because of a remarkable coincidence in the physical parameters governing light and motion propagation in these two materials, the equations for both longitudinal acoustic waves and normal-incidence light become practically equivalent for excitations of the same wavelength. This guarantees spatial overlap between the electromagnetic and displacement fields of specific photon-phonon pairs, leading to strong light-matter interaction. In particular, a statistical enhancement in the vacuum optomechanical coupling rate, $g_{o}$, is found, making this system a promising candidate to explore Anderson localization of high frequency $(\sim 20 \mathrm{GHz})$ phonons enabled by cavity optomechanics. The colocalization effect shown here unlocks the access to unexplored localization phenomena and the engineering of light-matter interactions mediated by Anderson-localized states.
\end{abstract}

DOI: 10.1103/PhysRevLett.122.043903

The engineering of wave scattering across all the frequency spectrum is at the heart of fundamental research in scientific disciplines dealing with objects spanning orders of magnitude in size. Scattering in the presence of disorder is pervasive in nature, with examples ranging from the diffusion of light as it passes through dust to the dynamics of sea waves in the presence of obstacles. A major breakthrough in wave scattering arises from the seemingly more inaccessible quantum-mechanical behavior of electrons in certain random atomic lattices [1]; diffusion halts when the scale of coherent multiple scattering is reduced to the wavelength itself, this being ascribed to the (Anderson) localization of the eigenmodes' wave functions. This transition was found to be inherent to any system governed by a wave equation in a disordered potential [2], prompting intense research activity [3-6]. Not suffering from mutual interactions as electrons do, electromagnetic radiation has concentrated much of the effort. Experimental attempts to observe Anderson localization of classical waves have often relied on the analysis of transport parameters [7-9], suffering from artifacts like absorption [10]. Alternatively, one can look at the relative size of the fluctuations in different transport parameters, an approach first proposed for microwave photons [11]. In optics, the far-field emission of excellent point sourcesi.e., quantum emitters-allows direct access to the statistics of Anderson localization and to spatially resolve the localized field [12]. For elastic waves, experiments have been so far restricted to ultrasound $[13,14]$ while the direct observation of localization at higher frequencies with explicit inspection of the individual localized wave fields simultaneously in the spatial and spectral domain remains elusive. The lack of practical phonon transitions in the solid state and their limited far-field radiation complicate the read-out and prevent the analogy to optics.

The interaction between electromagnetic radiation and mechanical motion in disordered periodic-on-average silicon nanobeams has been studied recently [15]. Since disorder localizes both fields, the confined optical modes can interact with the localized mechanical motion as in standard engineered cavity optomechanical systems [16]. These systems have proven to be suited to test fundamental quantum physics with macroscopic objects $[17,18]$ and are promising candidates as transducers in quantum systems [19] or ultrahigh sensitivity mass and force sensors [20], among many other applications [21-24]. When induced by 
disorder, they could be used as a fundamental local probe to observe Anderson localization of high frequency mechanical vibrations. Unfortunately, the electromagnetic and displacement fields interfere independently within a typical perturbed structure and hardly colocalize, thus hindering the maximum achievable values of the vacuum optomechanical coupling rate $g_{o}$ for such systems in the Andersonlocalization regime [15], generally making the read-out of motion via the optics unlikely. We circumvent this issue by using a certain type of epitaxially grown optical and acoustic superlattices based on GaAs and AlAs. Engineered defects in such a platform $[25,26]$ or their three-dimensional version in the form of micropillars [27,28] are attracting increasing interest for (very) high frequency cavity optomechanics. In particular, the GaAs/AlAs Fabry-Pérot resonators proposed in Ref. [25] are designed to optimally confine photons and to simultaneously behave as resonators for acoustic phonons with the same wavelength, quality factor, and field profile. Here, we explore this idea in random structures and show in our calculations that any multilayer composed by an arbitrary combination of these two materials exhibits almost the same mechanical and optical interference pattern even when induced by geometrical disorder. Subsequently, we propose GaAs/AlAs disordered multilayers as a cavityoptomechanical platform to enable observation of Anderson localization of mechanical excitations at frequencies so far unexplored, as well as a test bed to explore field-localization phenomena with coupled excitations.

To study the optical and acoustic properties of these superlattices, we use a transfer matrix formalism [29] (see Supplemental Material [30]). In this system, the solution to the continuum-mechanics equations in the absence of sources for longitudinal motion and to the Maxwell's equations at normal incidence can be solved with exactly the same formalism [34], provided that the acoustic impedances $Z_{i}$ are replaced by refractive indexes $n_{i}$ and the longitudinal acoustic velocities $v_{i}$ by the speed of light, $c / n_{i}$, in the different $i$ layers. For a succession of two different materials (1) and (2), for which the ratios $n_{1} / n_{2}=$ $Z_{1} / Z_{2}$ and $n_{1} / n_{2}=v_{2} / v_{1}$ then

$$
\mathbf{M}_{\mathrm{ac}}\left(\Omega_{m}\right)=\mathbf{M}_{\mathrm{op}}\left(K \Omega_{m}\right),
$$

where $\mathbf{M}_{\mathrm{ac}}\left(\Omega_{m}\right)$ and $\mathbf{M}_{\mathrm{op}}\left(\omega_{o}\right)$ are the transfer matrices connecting the acoustic and optical coefficients in the propagating-counterpropagating plane-wave basis between the incidence layer and the substrate and $K=\left(c / n_{1} / v_{1}\right)$ (see the Supplemental material [30]). Here, $\Omega_{m}\left(\omega_{o}\right)$ is used for the mechanical (optical) angular frequency of a time-harmonic solution. This ideal condition implies the exact equivalence of the optical and acoustic problems in the system. Using standard values [35] for the optical and mechanical coefficients for photons in the near-infrared and microwave phonons in $\operatorname{GaAs}(1)$ and $\operatorname{AlAs}(2)$, we have $n_{1} / n_{2}=1.193-1.199=Z_{1} / Z_{2}$ and
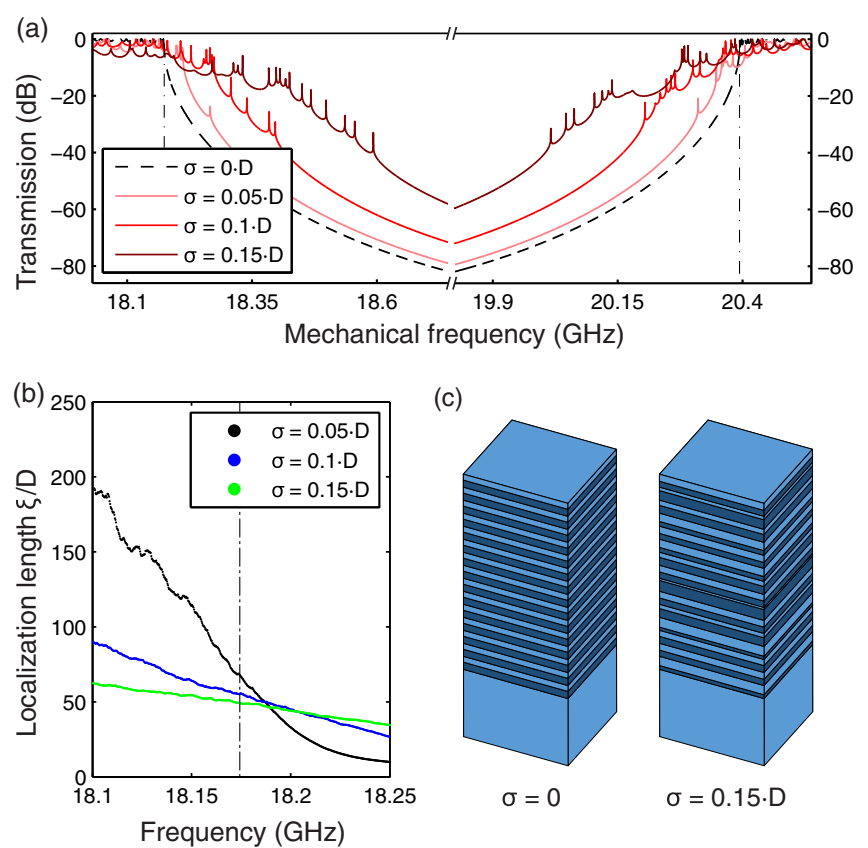

(c)

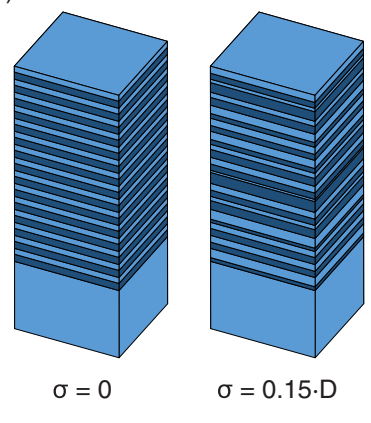

FIG. 1. Phonon localization in disordered GaAs/AlAs superlattices. (a) Transmission spectrum of perturbed GaAs/AlAs superlattices $\left(d_{1}=61.88 \mathrm{~nm}, d_{2}=73.48 \mathrm{~nm}, N=600\right)$, with Gaussian disorder $\mathcal{N}\left(d_{1}, \sigma^{2}\right)$ in the position of the interface inside the unit cell. (b) Localization length $\xi$ normalized by the unit cell thickness $D$ for several standard deviations $\sigma$. (c) Schematic of a perfect periodic superlattice $(\sigma=0)$ and a perturbed one $(\sigma=0.15 D)$ on a thick GaAs substrate.

$n_{1} / n_{2}=1.193-1.186=v_{2} / v_{1}$, which approximately satisfies the ideal conditions (1). Despite this slight departure from the ideal case, any arbitrary GaAs/AlAs superlattice exhibiting a mechanical eigenmode with a field profile $u(z)$ and angular frequency $\Omega_{m}$ will also support an optical eigenmode with angular frequency $\omega_{o} \sim K \Omega_{m}$ and field profile $E(z) \sim u(z)$ as shown in this Letter. To illustrate this, in our calculations we use a distributed Bragg reflector (DBR) structure with a unit cell formed by a GaAs layer of thickness $d_{1}=61.88 \mathrm{~nm}$ and an AlAs layer of thickness $d_{2}=73.48$ (technically known as a $\lambda / 4, \lambda / 4$ DBR). This structure simultaneously opens a maximum-width firstorder band gap with a center frequency of $344.6 \mathrm{THz}$ $(\lambda=870 \mathrm{~nm})$ for near infrared photons and $19 \mathrm{GHz}$ for microwave phonons. We introduce geometrical disorder in the position of the interface between the two materials. Its natural position at $z=d_{1}$ is Gaussian distributed $\mathcal{N}\left(d_{1}, \sigma^{2}\right)$ with varying standard deviation $\sigma$, while the period $D=$ $d_{1}+d_{2}=135.36 \mathrm{~nm}$ is kept constant. Figure 1 shows the acoustic transmission spectrum of a disordered superlattice with $N=600$ periods for different disorder levels, $\sigma$, around the first-order odd band gap.

Noninteracting electronic Bloch modes in atomic crystals undergo random multiple scattering in the presence of disorder, eventually leading to the (Anderson) localization 

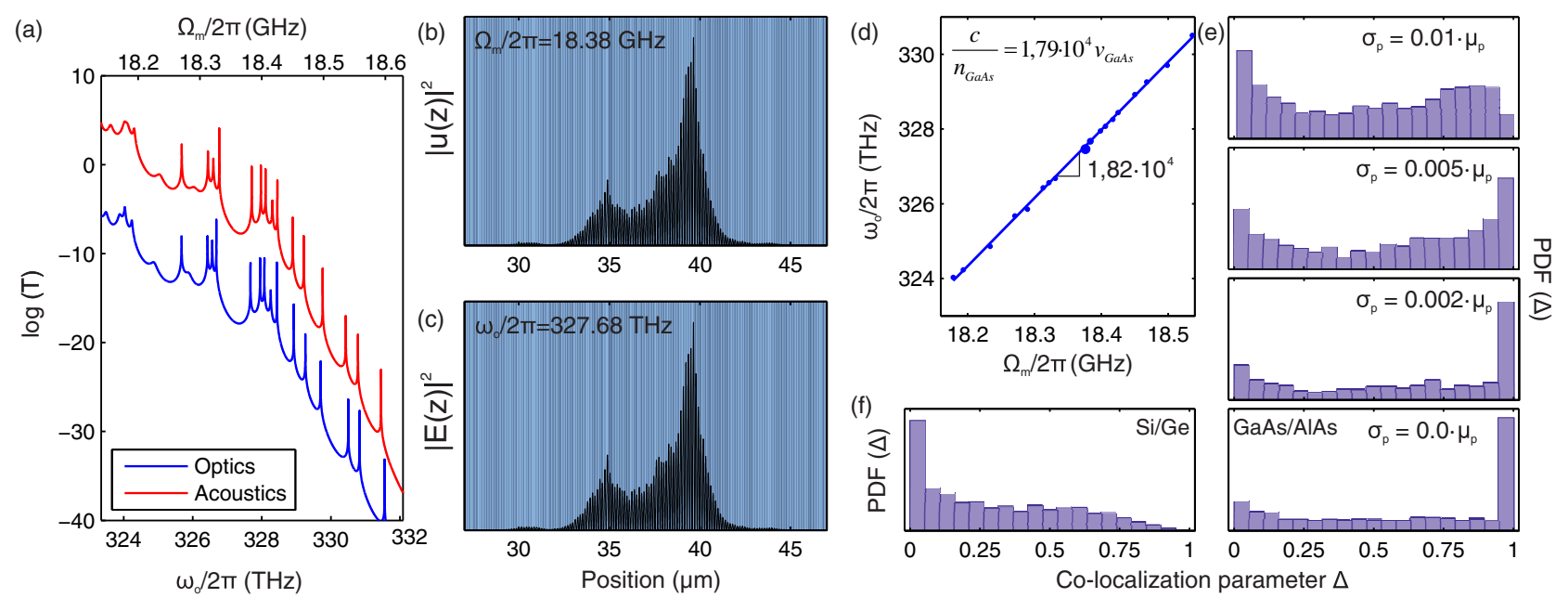

FIG. 2. Photon-phonon colocalization. (a) Optical and acoustic transmission spectra of a disordered GaAs/AlAs superlattice with $d_{1}=61.88 \mathrm{~nm}, d_{2}=73.48 \mathrm{~nm}, N=600$, and $\sigma=0.15 \mathrm{D}$ at the low-frequency optical and mechanical band-gap edge, respectively. The energy density of the displacement field $u(z)$ and the electric field $E(z)$ of the highlighted pair in (d) are plotted in (b) and (c), showing perfect spatial colocalization. The resonant frequencies of all the mechanical and optical eigenmodes are mapped onto each other and plotted in (d). (e) Histogram of $\Delta$ for $2 \times 10^{3}$ disordered GaAs/AlAs superlattices with their physical parameters $\{\rho, v, n\}$ normally distributed with a standard deviation $\sigma_{p}$. (f) Histogram of the colocalization parameter $\Delta$ for a set of $2 \times 10^{3}$ disordered $\mathrm{Si} / \mathrm{Ge}$ superlattices.

of the wave function [1]. The electromagnetic and displacement fields in artificial photonic and phononic crystals are also sensitive to disorder, especially at the band-gap edges where the group velocity falls (ideally) to 0 [6], equally giving rise to disorder-induced or Anderson localization. In a one-dimensional structure such as a GaAs/AlAs superlattice, the presence of Anderson-localized modes can be detected in the reflection or transmission spectrum through Lorentzian-shaped resonances centered near the corresponding eigenfrequencies with a free spectral range $\delta \omega$ larger than the mean linewidth $\Delta \omega$ [36]. Figure 1(a) shows these narrow mechanical resonances in the transmission spectra visibly satisfying such criteria and populating a spectral band that broadens with increasing disorder level [37]. The ensemble-averaged decay of such localized modes occurs with a localization length, $\xi$. It determines the minimum length of a finite sample for which it is possible to statistically observe these modes as $L \geq \xi$ [38], where $L=N D$ is the total length of the structure. Figure 1(b) shows $\xi$ as a function of disorder level and frequency, using the scaling of the ensemble-averaged logarithmic transmission $\langle\log (T)\rangle \propto-(L / \xi)$ and evidences that the system is deep in the localization regime $(\xi \ll L)$ near the band edges for the range of disorders and lengths $(N=600)$ considered later in this Letter.

When condition (1) is fulfilled, the optical spectrum is almost identical to the mechanical one when plotted with scaled frequency, as shown in Fig. 2(a). When the mechanical and optical resonant frequencies are mapped onto each other in spectral order, we recover [Fig. 2(d)] the predicted spectral behavior with $\omega_{o} \approx C \Omega_{m}$ and
$C=1.82 \times 10^{4}-1.79 \times 10^{4}=K$. To calculate the optical $\left\{\omega_{n}, E_{n}(z)\right\}$ and the mechanical $\left\{\Omega_{m}, u_{m}(z)\right\}$ eigenmodes, we apply the transfer matrix method with outgoing boundary conditions and solve for the complex eigenfrequencies $\omega=\omega_{r}-j \omega_{i}$ of the quasinormal modes [39] (see details in Supplemental Material [30]). For the highlighted photonphonon pair of the structure simulated in Fig. 2(d), Figs. 2(b) and 2(c) show how the displacement and electric field intensities respectively are also perfectly overlapping in real space. In order to quantify the degree of spatial colocalization we define the parameter

$$
\Delta_{n m}=\frac{\int\left|u_{m}(z)\right|\left|E_{n}(z)\right| d z}{\sqrt{\int\left|u_{m}(z)\right|^{2} d z \int\left|E_{n}(z)\right|^{2} d z}},
$$

where $\Delta \in[0,1]$ with $\Delta=1$ being fully colocalized. Here $u_{m}(z)$ is the mechanical displacement and $E_{n}(z)$ the electric field properly normalized by the amplitude of the field in the substrate, and the amplitude of the incident plane wave from the air, respectively. We calculate $\Delta$ between all the photonic and phononic modes in a narrow frequency range around the lower band edge from a set of $2 \times 10^{3}$ GaAs/AlAs superlattices with the same structural parameters as detailed above and the disorder level fixed at $\sigma=0.15 D$. The histogram of $\Delta$, plotted in the lowest panel in Fig. 2(e), is strongly peaked for $\Delta>0.95$ for which the modes are perfectly colocalized and presents rather low values for the $\Delta<0.95$. To verify the robustness of this significant colocalization effect, we calculate the distribution of $\Delta$ while adding additional Gaussian disorder to the 
material parameters $\{\rho, v, n\}$ of both GaAs and AlAs, with standard deviations $\sigma_{p}=[0.002,0.005,0.01] \cdot \mu_{p}, \quad \mu_{p}$ being the mean value for the given physical parameter. As plotted in the upper panel in Fig. 2(e), a disorder level larger than $\sigma_{p}=0.01 \mu_{p}$ is required to fully wipe out the effect, well above the layer-by-layer statistical fluctuations achievable in actual molecular-beam epitaxy-grown samples [40] (see details in Supplemental Material [30]). For comparison, we calculate the distribution of $\Delta$ for a $\mathrm{Si} / \mathrm{Ge}$ disordered superlattice. The $\mathrm{Si}$ and $\mathrm{Ge}$ thicknesses $\left(d_{1}=115.97 \mathrm{~nm}, d_{2}=93.88 \mathrm{~nm}, N=600\right)$ are chosen to tune the band edge of interest at approximately half the frequency of the GaAs/AlAs superlattice to avoid the effect of absorption. The disorder level is also shifted to $\sigma=$ $0.11 D$ to satisfy the condition $\xi_{\mathrm{Si} / \mathrm{Ge}} \sim \xi_{\mathrm{GaAs} / \mathrm{AlAs}}$. In this case, the distribution $\Delta$ [Fig. 2(f)] shows no colocalization at all.

These well-confined spatially overlapping modes can interact with each other and are therefore candidates to explore their coupling. As expected from confined mechanical and light modes, the deformation profile associated with a normal mode $u_{m}(z)$ will locally change the optical properties of the structure. Thus, the electromagnetic eigenmodes $E_{n}(z)$ will be affected, giving rise to both an optical frequency shift (dispersive interaction) and a quality factor change (dissipative interaction). In $\mathrm{GaAs} / \mathrm{AlAs}$ superlattices and in the frequency range of interest, mainly two acousto-optic interaction mechanisms need to be considered [41,42]. First, the displacement of the $N+1$ boundaries, or moving boundary effect, will change the interference pattern of multiple light paths. Second, the photoelastic effect will induce a change in the bulk permittivity tensor $\epsilon$ that can be written as the tensor product $d\left(\epsilon^{-1}\right)_{i j}=P_{i j k l} U k l$, where $\mathbf{U}$ is the second-order strain tensor and $\mathbf{P}$ the fourth-order photoelastic tensor [43]. To account for the frequency shift induced by these two mechanisms, first-order perturbation theory applied to Maxwell's equations [44] in the multilayered system leads to the following expressions:

$g_{m b}=-\frac{\omega_{o}}{2} \frac{\sum_{i=1}^{N+1} u_{m}\left(z_{i}\right)\left(\epsilon_{i-1}-\epsilon_{i}\right)\left|E_{n}\left(z_{i}\right)\right|^{2}}{\int_{0}^{L} \epsilon(z)\left|E_{n}(z)\right|^{2} d z} x_{z p f}$,

$g_{p e}=-\frac{\omega_{o}}{2} \frac{\int_{L}^{0} P_{12}(z) \frac{\partial u_{m}}{\partial z}(z) \epsilon(z)^{2}\left|E_{n}(z)\right|^{2}}{\int_{0}^{L} \epsilon(z)\left|E_{n}(z)\right|^{2} d z} x_{z p f}$.

The strength of the coupling parameter $g_{o}=g_{m b}+g_{p e}$ between two ( $n$-optical, $m$-mechanical) eigenmodes strongly depends on the overlap of the displacement $u_{m}(z)$ or strain $\left[\partial u_{m} / \partial z\right](z)$ field with the electric field intensity $\left|E_{n}(z)\right|^{2}$, which highlights the importance of colocalizing the displacement and the electric field profiles. Since our model is by definition one dimensional, we have chosen an arbitrary size in the $x-y$ plane of $2 \times 2 \mu \mathrm{m}^{2}$, for


FIG. 3. Vacuum optomechanical coupling rate $g_{o} / 2 \pi$ in disordered multilayered systems. (a) Scatter plot of the mechanical and optical frequencies of the resonant modes found in a set of $2 \times 10^{3}$ disordered GaAs/AlAs multilayers $(\sigma=0.15 D)$; the color represents the coupling rate $g_{o} / 2 \pi$ between the considered pair in logarithmic scale. (b) The upper panel plots the histogram of the coupling rate $g_{o} / 2 \pi$ considering poorly colocalized photon-phonon pairs $(\Delta<0.95$, blue bins $)$ and only perfectly colocalized pairs ( $\Delta>0.95$, red bins). The bottom panel plots the histogram of the coupling rate $g_{\text {mod }} / 2 \pi$ defined in an inset. The corresponding dashed lines give the value of $g_{o} / 2 \pi$ and $g_{\bmod } / 2 \pi$ expected for a perfect Fabry-Pérot cavity based on two unperturbed DBR mirrors of 300 cells each.

the area excited by a focused laser beam, in order to calculate the effective mass $m_{\mathrm{eff}}$ of the mechanical resonator and the zero point fluctuations $x_{z p f}=\sqrt{\hbar / 2 m_{\mathrm{eff}} \boldsymbol{\Omega}_{m}}$. The product of the coupling integral by $x_{z p f}$ quantifies the interaction between a single phonon and a single photon, and is required since otherwise the parameter would depend on the normalization chosen for $u_{m}(z)$.

We evaluate $g_{o}$ between the optical and mechanical modes of the GaAs/AlAs disordered superlattices simulated for Fig. 2(e). Because of the strongly dispersive nature of the localization length $\xi$ [Fig. 1(b)], we consider only a narrow frequency window, thus minimizing the effect of varying the effective volume of the eigenmodes. The coupling values in the densely packed diagonal of Fig. 3(a), i.e., for perfectly colocalized photon-phonon pairs $(\Delta>0.95)$, exhibit values higher than the poorly colocalized ones $(\Delta<0.95)$. The probability density function of the vacuum optomechanical coupling rate $g_{o}$ 
is plotted in Fig. 3(b) either considering poorly colocalized modes (blue bars), or those perfectly colocalized (red bars), pointing out a statistical evidence for this material combination. The coupling mechanism considered in (3b) is based on the product of the oscillating function $\left(\partial u_{m} / \partial z\right)(z)$ by the always positive $\epsilon(z)^{2}\left|E_{n}(z)\right|^{2}$. When two fields $u_{m}(z)$ and $E_{n}(z)$ are perfectly colocalized, the function $\left(\partial u_{m} / \partial z\right)(z), \pi / 2$-shifted version of $u_{m}(z)$, is null whenever $\left|E_{n}(z)\right|^{2}$ attains a maximum, and vice versa for the maxima or minima of $\left(\partial u_{m} / \partial z\right)(z)$. Despite the perfect coincidence of the fields, the integrand values stay low, since $\epsilon(z)^{2}$ and $p_{12}(z)$ have their own particular spatial dependence. As a consequence, partially overlapping photon-phonon pairs may present similar couplings as perfectly overlapping ones, which reduces the effect of colocalization for this particular photoelastic coupling when compared to other light-matter processes. To emphasize the general interest of the colocalization effect, we calculate the effect of colocalization on different type of light-matter processes, i.e., with different functional form of the parametric coupling (see Supplemental Material [30]). In particular, Fig. 3(c) plots the histogram of the coupling rates considering the energy intensities $\left|E_{n}(z)\right|^{2}$ and $\left|u_{m}(z)\right|^{2}$ of the optical and mechanical eigenmodes while $p_{12}(z)$ remains fixed, which shows the potential of the photon-phonon colocalization induced in this system. For comparison, we calculate the different couplings discussed here for a perfect Fabry-Pérot cavity formed by two 300 cell DBR mirrors, highlighted with dashed lines in the panels of Fig. 3(b).

In conclusion, we present an analysis of simultaneous Anderson localization of two wave fields in a particular set of random superlattices, with emphasis on their spatial overlap and their acoustooptic interaction. Our calculations provide quantitative evidence of the role played by spatial colocalization of mechanical and optical excitations in GaAs/AlAs superlattices in which the likelihood of colocalization is greatly enhanced. As a consequence, the coupling of the light field to motion in GaAs/AlAs DBR-based cavity structures appears to be a natural and appropriate choice to observe Anderson localization of phonons in frequency ranges so far unexplored, and even to explore Anderson localization of coupled excitations [45]. Moreover, due to the optomechanical nature of standard phonon generation or detection experiments in GaAs/AlAs mutilayers [46], the conditions for read-out are well understood. The integration of quantum emitters [47] during molecular-beam epitaxy growth could enable cavity quantum-electrodynamics experiments in the Andersonlocalization regime coupled to the motion of high frequency nanomechanical oscillators, as well as to explore the role of phonon-mediated dephasing in random lasers [48]. Finally, the discussed structures can be scaled down to study Anderson localization of sub-THz vibrations with extended light modes.
This work was supported by the Spanish Ministerio de Ciencia, Innovacion y Universidades (MICINN) via the Severo Ochoa Program (Grant No. SEV-2017-0706) and the project PHENTOM (Grant No. Fis 2015-70862-P) as well as by the Centres de Recerca de Catalunya (CERCA), and by the European Commission in the form of the H2020 FET open project PHENOMEN (Grant No. 713450). G. A. is supported by a Barcelona Institute of Science and Technology PhD fellowship, N.D.L.K. by the ERC Starting Grant Nanophennec (Grant No. 715939) and P. D. G. by a Ramon y Cajal fellowship (Grant No. RyC-2015-18124).

*guillermo.arregui@icn2.cat david.garcia@icn2.cat

[1] P. W. Anderson, Phys. Rev. 109, 1492 (1958).

[2] F. Evers and A. D. Mirlin, Rev. Mod. Phys. 80, 1355 (2008).

[3] J. Jäckle, Solid State Commun. 39, 1261 (1981).

[4] P. E. Wolf and G. Mazret, Phys. Rev. Lett. 55, 2696 (1985).

[5] M. P. Van Albada and A. Lagendijk, Phys. Rev. Lett. 55, 2692 (1985).

[6] S. John, Phys. Rev. Lett. 58, 2486 (1987).

[7] N. Garcia and A. Z. Genack, Phys. Rev. Lett. 66, 1850 (1991).

[8] D. S. Wiersma, P. Bartolini, A. Lagendijk, and R. Righini, Nature (London) 390, 671 (1997).

[9] F. Scheffold, R. Lenke, R. Tweer, and G. Maret, Nature (London) 398, 206 (1999).

[10] S. E. Skipetrov and J. H. Page, New J. Phys. 18, 021001 (2016).

[11] A. A. Chabanov, M. Stoytchev, and A. Z. Genack, Nature (London) 404, 850 (2000).

[12] P. D. García and P. Lodahl, Ann. Phys. (Amsterdam) 529, 1600351 (2017).

[13] R. L. Weaver, Wave Motion 12, 129 (1990).

[14] H. Hu, A. Strybulevych, J. H. Page, S. E. Skipetrov, and B. A. van Tiggelen, Nat. Phys. 4, 945 (2008).

[15] P. D. García, R. Bericat-Vadell, G. Arregui, D. NavarroUrrios, M. Colombano, F. Alzina, and C. M. SotomayorTorres, Phys. Rev. B 95, 115129 (2017).

[16] M. Aspelmeyer, T. J. Kippenberg, and F. Marquardt, Rev. Mod. Phys. 86, 1391 (2014).

[17] E. Verhagen, S. Deléglise, S. Weis, A. Schliesser, and T. J. Kippenberg, Nature (London) 482, 63 (2012).

[18] A. D. O’Connell, M. Hofheinz, M. Ansmann, R. C. Bialczak, M. Lenander, E. Lucero, M. Neeley, D. Sank, H. Wang, M. Weides, J. Wenner, J. M. Martinis, and A. N. Cleland, Nature (London) 464, 697 (2010).

[19] R. A. Norte, J. P. Moura, and S. Gröblacher, Phys. Rev. Lett. 116, 147202 (2016).

[20] E. Gavartin, P. Verlot, and T. J. Kippenberg, Nat. Nanotechnol. 7, 509 (2012).

[21] A. G. Krause, M. Winger, T. D. Blasius, Q. Lin, and O. Painter, Nat. Photonics 6, 768 (2012).

[22] S. Weis, R. Rivière, S. Deléglise, E. Gavartin, O. Arcizet, A. Schliesser, and T. J. Kippenberg, Science 330, 1520 (2010). 
[23] J. Rosenberg, Q. Lin, and O. Painter, Nat. Photonics 3, 478 (2009).

[24] J. T. Hill, A. H. Safavi-Naeini, J. Chan, and O. Painter, Nat. Commun. 3, 1196 (2012).

[25] A. Fainstein, N. D. Lanzillotti-Kimura, B. Jusserand, and B. Perrin, Phys. Rev. Lett. 110, 037403 (2013).

[26] P. Sesin, P. Soubelet, V. Villafañe, A. E. Bruchhausen, B. Jusserand, A. Lemaître, N. D. Lanzillotti-Kimura, and A. Fainstein, Phys. Rev. B 92, 075307 (2015).

[27] F. R. Lamberti, Q. Yao, L. Lanco, D. T. Nguyen, M. Esmann, A. Fainstein, P. Sesin, S. Anguiano, V. Villafañe, A. E. Bruchhausen, P. Senellart, I. Favero, and N.D. Lanzillotti-Kimura, Opt. Express 25, 24437 (2017).

[28] S. Anguiano, A. E. Bruchhausen, B. Jusserand, I. Favero, F. R. Lamberti, L. Lanco, I. Sagnes, A. Lemaître, N. D. Lanzillotti-Kimura, P. Senellart, and A. Fainstein, Phys. Rev. Lett. 118, 263901 (2017).

[29] S. M. Rytov, Akust. Zh. 2, 71 (1956).

[30] See Supplemental Material at http://link.aps.org/ supplemental/10.1103/PhysRevLett.122.043903 for a detailed description of the transfer matrix formalism used and the study of the robustness of the colocalization phenomena, which includes Refs. [31,33].

[31] N. D. Lanzillotti-Kimura, A. Fainstein, and B. Jusserand, Ultrasonics 56, 80 (2015).

[32] J.-P. Berenger, J. Comput. Phys. 114, 185 (1994).

[33] E. Anemogiannis and E. N. Glytsis, J. Lightwave Technol. 10, 1344 (1992).

[34] M. Born and E. Wolf, Principles of Optics: Electromagnetic Theory of Propagation, Interference and Diffraction of Light, 1st ed. (Pergamon Press, Oxford, 1964).
[35] S. Adachi, GaAs and Related Materials: Bulk Semiconducting and Superlattice Properties (World Scientific Publishing, Singapore, 1994).

[36] P. W. Anderson, D. J. Thouless, E. Abrahams, and D. S. Fisher, Phys. Rev. B 22, 3519 (1980).

[37] I. M. Lifshitz, Adv. Phys. 13, 483 (1964).

[38] P. Sheng, Introduction to Wave Scattering, Localization, and Mesoscopic Phenomena (Academic Press, San Diego, 1995).

[39] M. Maksimovic, M. Hammer, and E. van Groesen, Opt. Commun. 281, 1401 (2008).

[40] S. Gehrsitz, F. K. Reinhart, C. Gourgon, N. Herres, A. Vonlanthen, and H. Sigg, J. Appl. Phys. 87, 7825 (2000).

[41] P. Ruello and V. E. Gusev, Ultrasonics 56, 21 (2015).

[42] O. Matsuda and O. B. Wright, Ultrasonics 40, 753 (2002).

[43] O. Matsuda and O. B. Wright, J. Opt. Soc. Am. B 19, 3028 (2002).

[44] S. G. Johnson, M. Ibanescu, M. A. Skorobogatiy, O. Weisberg, J. D. Joannopoulos, and Y. Fink, Phys. Rev. E 65, 066611 (2002).

[45] T.F. Roque, Vi. Peano, O. M. Yevtushenko, and F. Marquardt, New J. Phys. 19, 013006 (2017).

[46] N. D. Lanzillotti-Kimura, A. Fainstein, A. Lemaître, B. Jusserand, and B. Perrin, Phys. Rev. B 84, 115453 (2011).

[47] T. Czerniuk, C. Brüggemann, J. Tepper, S. Brodbeck, C. Schneider, M. Kamp, S. Höfling, B. A. Glavin, D. R. Yakovlev, A. V. Akimov, and M. Bayer, Nat. Commun. 5, 4038 (2014).

[48] J. Liu, P. D. García, S. Ek, N. Gregersen, T. Suhr, M. Schubert, J. Mørk, S. Stobbe, and P. Lodahl, Nat. Nanotechnol. 9, 285 (2014). 